Authors' reply: We welcome the responses by Cantor and Nielssen \& Large to our editorial. On the lighter side, we observe that yet more proposals for post-traumatic conditions are proposed by Cantor (e.g. PSRHD), thereby demonstrating an ever increasing incidence of 'acronymitis'. This disorder, characterised by a seeming compulsion to develop acronyms, was to the best of our knowledge first labeled by Isaac Marks (personal communication, 2005).

On a more serious note, we would like to use our limited space to highlight several observations that we have taken from an extensive review of the PTSD construct. ${ }^{1}$ This review proposes that PTSD's defined clinical syndrome might best be conceptualised as encompassing a broad range of reactions to adverse events that are in turn influenced by multiple dimensionally distributed factors (e.g. pre- and post-incident risk variables, peri-traumatic appraisals and real-life consequences). The long history of general stress studies, and more recent research on PTSD, has demonstrated that these multiple factors and their complex interrelations yield a wide range of outcomes after adverse events. Within this framework, it remains an open question whether any attempt to define a distinct post-traumatic syndrome can lead to a true disorder in nature that is specific to a subset of stressors. Perhaps such a disorder exists, and PTSD or some other acronym should remain in the psychiatric nomenclature. For the moment, however, it appears that the very literature spurred by the creation of PTSD has demonstrated, somewhat ironically, that the construct is flawed. It is in the context of these concerns that we acknowledge issues raised in the Journal's letters and encourage continued discussion on the validity of the PTSD diagnosis.

1 Rosen GM, Lilienfeld SO. Posttraumatic stress disorder: an empirical analysis of core assumptions. Clin Psychol Rev 2007; in press.

Gerald M. Rosen, University of Washington, Seattle, Washington, USA. Email: grosen@u.washington.edu; Robert L. Spitzer, Columbia University, New York, USA Paul R. McHugh, Johns Hopkins University School of Medicine, Baltimore, Maryland, USA

doi: 10.1192/bjp.192.5.395

\section{Lithium in mood disorders: a one-sided re-appraisal}

To the uncritical mind, it appears as if Young \& Hammond ${ }^{1}$ have made a case for more use of lithium in mood disorders than is currently the trend. They partly based their argument on the meta-analysis by Smith et al. ${ }^{2}$ A close perusal of the meta-analysis, however, revealed that the case made by Young \& Hammond for lithium is one-sided, unbalanced and may be misleading. Even though the study by Smith et al stated that lithium remains the medication with the strongest evidence base, we believe that its declining use may be due to incontrovertible evidence of adverse effects. For example, in the meta-analysis by Smith et al, when withdrawals for any reason and withdrawals for adverse events were analysed, there were more withdrawals with lithium compared with lamotrigine, valproate semisodium and olanzapine. Even in terms of efficacy, the choice of lithium remains arguable. For example, when relapses due to depression were analysed, Smith et al found that there were more relapses with lithium than with lamotrigine and valproate semisodium. In terms of manic episode, there were more relapses with lithium compared with olanzapine, and in terms of any mood episode, there were more relapses with lithium than valproate semisodium and olanzapine.

We do not advocate for any particular medication but we strongly feel that for this type of medication advocacy, authors should attempt to provide a balanced rather than one-sided argument. It is also patronising to partly ascribe the declining use of lithium to poor training of psychiatrists rather than acknowledge the fact that psychiatrists may actually base their choices on individual patient criteria as well as the profile of medications within the wide array of available agents.

1 Young $\mathrm{AH}$, Hammond $\mathrm{MH}$. Lithium in mood disorders: increasing evidence base, declining use? Br J Psychiatry 2007; 191: 474-6.

2 Smith LA, Cornelius V, Warnock A, Bell A, Young AH. Effectiveness of mood stabilizers and antipsychotics in the maintenance phase of bipolar disorder: a systematic review of randomized controlled trials. Bipolar Disord 2007; 9 : 394-412.

Babatunde Adetunji, New York University Medical Center, Manhattan, New York, USA. Email: medikhelp@yahoo.com; Oluyemisi Adetunji, Steininger Behavioral Health Care Services, Cherry Hill, New Jersey, USA; Andrew Newton, Mount Carmel Neuropsychiatric Consultants, Mount Carmel, Pennsylvania, USA

doi: 10.1192/bjp.192.5.395a

Authors' reply: We are pleased that Adetunji et al read our paper and saw fit to comment upon it. However, we are surprised at the nature of their remarks, which suggest that not only did they not read our piece with particular care, they have perhaps also not thoroughly read (or perhaps understood) the paper they quote by Smith et al. ${ }^{1}$ One of us (A.H.Y.) is the senior and corresponding author on this meta-analysis and thus very familiar with the content! Close perusal of our meta-analysis does not show our case for lithium to be one-sided, unbalanced and misleading. Indeed, we conclude 'mood stabilisers have differing profiles of efficacy and tolerability' and demonstrate that lithium has clear evidence of both tolerability and efficacy. Nowhere do we suggest that lithium is the best treatment for every patient with bipolar disorder, nor is the purpose of the article to review the evidence for all bipolar medications. Rather, as we state in our conclusion, our argument is that lithium remains the best treatment in a significant portion of cases and must be included in any psychiatrist's treatment arsenal. The reason this message is so important is that lithium is increasingly being neglected as a treatment option in several countries, resulting in inadequate treatment of some patients with the disorder, who are then labelled 'treatment-resistant' without having ever tried lithium. ${ }^{2}$

Prescribing patterns are influenced by pharmaceutical company promotion - or why would companies spend this money? ${ }^{3}$ Jefferson ${ }^{4}$ and $\mathrm{Chan}^{5}$ both report declining psychiatry resident knowledge about, and use of, older medications (including lithium) despite evidence supporting their continued use; we are unclear why Adetunji et al find this literature 'patronising'. We agree with them, however, that psychiatrists should base treatment choices on individual patient characteristics as well as the profile of medicines. Applying this approach to the wide array of available agents will undoubtedly ensure that lithium continues to be widely used for the foreseeable future.

1 Smith LA, Cornelius V, Warnock A, Bell A, Young AH. Effectiveness of mood stabilizers and antipsychotics in the maintenance phase of bipolar disorder: a systematic review of randomized controlled trials. Bipolar Disord 2007; 9: 394-412.

2 Jefferson JW. Rediscovering the art of lithium therapy. Curr Psychiatry 2002; 1: 19-24.

3 Huang FY, Weiss DS, Fenimore PG, Fleming AM, Haller E, Lichtmacher JE, Eisendrath SJ. The association of pharmaceutical company promotional spending with resident physician prescribing behavior. Acad Psychiatry 2005; 29: $501-2$.

4 Jefferson JW. Old versus new medications: how much should be taught? Acad Psychiatry 2005; 29: 162-6.

5 Chan $\mathrm{CH}$. The pharmaceutical role. Acad Psychiatry 2006; 30: 45-7.

Allan H. Young, Department of Psychiatry, University of British Columbia, Vancouver, British Columbia, Canada V6T 1Z3. Email: allanyoun@gmail.com; Judith M. Hammond, Department of Psychiatry, University of British Columbia, Vancouver, British Columbia, Canada

doi: $10.1192 /$ bjp.192.5.395b 